\title{
Erratum to: Processing of joint molecule intermediates by structure-selective endonucleases during homologous recombination in eukaryotes
}

\author{
Erin K. Schwartz • Wolf-Dietrich Heyer
}

Published online: 20 May 2011

(C) Springer-Verlag 2011

\section{Erratum to: Chromosoma \\ DOI 10.1007/s00412-010-0304-7}

The original version of this article unfortunately contained some mistakes.

In Figure 1, the legend contains a mistake. The corrected figure legend is given below.

Multiple DNA repair pathways are employed during doublestrand break repair. After DNA DSB formation, the broken ends can be religated using minimal nucleotide homology through nonhomologous end joining (NHEJ). Alternatively, 5' ends are resected to expose single-stranded DNA favoring alternate routes of DSB repair. The presence of direct DNA sequence repeats may provide homologous regions that can anneal to form a contiguous chromosome in a process called single-strand annealing (SSA). Heterologous 3' flaps are removed by the $\mathrm{XPF}$ endonuclease aided by Slx 4 and Saw1. Alternatively, Rad51-dependent homology search and strand invasion forms a displacement loop (D-loop) to prime DNA synthesis from the $3^{\prime}-\mathrm{OH}$ end of the broken chromosome on an intact template. Extension of the D-loop and subsequent D-loop disruption and reannealing to the second end repairs the break via synthesis-dependent strand annealing (SDSA) resulting in noncrossover (NCO) products (Resnick 1976). Formation of an intact replication fork leads to the continuous extension of the D-loop to the end of the chromosome, defining the breakinduced replication (BIR) pathway and resulting in loss of heterozygosity (LOH) (Malkova et al. 1996). The elongated D-loop forms a junction, where branch migration may lead to the formation of a single Holliday junction (sHJ). In the event of second-end capture, the displaced strand of the D-loop anneals to the other resected $3^{\prime}$ strand forming first two nicked Holliday junctions (nHJ) and after ligation a double Holliday junction (dHJ) (Szostak et al. 1983). Double HJs can be dissolved by the combined activities of a DNA motor protein (S. cerevisiae Sgs1 or human BLM) and a type IA topoisomerase into NCO products (Wu and Hickson 2003; Cejka et al. 2010) or resolved by coordinated endonuclease cleavage into CO or NCO products (Szostak et al. 1983). Single HJs require resolution by anuclease and cannot be processed by a dissolution mechanism like dHJs.

The online version of the original article can be found at http:/dx.doi. org/10.1007/s00412-010-0304-7.

E. K. Schwartz $(\bowtie) \cdot$ W.-D. Heyer

Department of Microbiology, University of California-Davis,

Davis, CA 95616-8665, USA

e-mail: wdheyer@ucdavis.edu 
Table 1 contains 4 typographical errors, see the corrected table below.

Table 1 Structure-selective endonucleases exhibit an array of species specific differences. Organized by a single identifying subunit, eukaryotic homologs are listed with species designation. Information on their known binding partners, respective endonuclease superfamily, and mechanistic pathway involvement are provided

\begin{tabular}{|c|c|c|c|c|}
\hline Indenfifying subunit & Protein complex & Onuclease farown & In vivo function & References \\
\hline \multirow[t]{5}{*}{ Radl } & ScRadl -RadiO & $\mathrm{XPF}$ & NER, ICL, SSA & $\begin{array}{l}\text { (Cox and Parry 1968; Fishman } \\
\text { Lobell et al. 1992) }\end{array}$ \\
\hline & SpRadl6-Swi10 & XPF & NER, MTS & (Carr et at 1994; Schmidt et at. 1989) \\
\hline & AtRAD1-RAD1O & XPF & NER, ICL, SSA & (Gallego at al. 2000; Duhest et al. 2002) \\
\hline & DmMEI-9-ERCCI & XPF & NER, ICL, Meiosis & (Redford et at. 2005) \\
\hline & $H_{s} \mathrm{XPF}(\mathrm{EROD} 4)-\mathrm{ERCC} 1$ & $\mathrm{XPF}$ & NER, ICL & $\begin{array}{l}\text { (Biggerstaff et al. 1993; van } \\
\text { Vuuren et al. 1993) }\end{array}$ \\
\hline \multirow[t]{5}{*}{ Mus81 } & ScMus81-Mms4 & XPF & HR, RF, Meiosis ICL & (Interthal and Heyer 2000) \\
\hline & SpMus81-Eme1 & $\mathrm{XPF}$ & HR, RF, Meiosis & (Boddy et at. 2000) \\
\hline & ATMUS81-EMEA/EMEB & XPF & HR, RP & (Berchowitz et al. 2007) \\
\hline & DmMUS81-EME1 & $\mathrm{XPF}$ & HR & $\begin{array}{l}\text { (Johnson-Schlitz and Engels 2006; } \\
\text { Trowbridge et al. 2007) }\end{array}$ \\
\hline & $H s / M m$ MUS81-EME1 & XPF & HR,ICL & (Abraham et al. 2003) \\
\hline \multirow[t]{4}{*}{ Yenl } & ScYenl & $\operatorname{Rad} 2 / X P G$ & $\mathrm{~N} / \mathrm{D}$ & \\
\hline & $D m$ GEN & $\operatorname{Rad} 2 / X P G$ & $\mathrm{~N} / \mathrm{D}$ & \\
\hline & CeGEN-1 & $\operatorname{Rad} 2 / X P G$ & DSBR & (Bailly et al. 2010) \\
\hline & $H s \mathrm{GEN} 1$ & $\operatorname{Rad} 2 / X P G$ & $\mathrm{~N} / \mathrm{D}$ & \\
\hline \multirow[t]{15}{*}{ Slx 4} & ScSlx4 complexes & & & \\
\hline & Radl-Rad10-S1x4 & XPF & SSA & (Flott et al. 2007) \\
\hline & Slx $1-S 1 x 4$ & UIY-YIG & rDNA & (Kaliraman and Brill 2002) \\
\hline & SpSlx 4 complexes & & & \\
\hline & Slxl-S1x4 & UIY-YIG & rDNA & (Coulon et al. 2004) \\
\hline & DmMUS312 complexes & & & \\
\hline & MEI-9-ERCC1?-MUS312 & XPF & NER, ICL, Meiosis & (Yildiz et al. 2002) \\
\hline & SLX1-MUS312 & UIY-YIG & $\mathrm{N} / \mathrm{D}$ & \\
\hline & CeHIM-18 complexes & & HR, RF, DSBR, Meiosis & Saito et al. 2009) \\
\hline & $X P F$-ERCC1?-HIM-18 & XPF & $\mathrm{N} / \mathrm{D}$ & \\
\hline & SLX1-HIM-18 & UIY-YIG & $\mathrm{N} / \mathrm{D}$ & \\
\hline & HsBTBD12 complexes & & & \\
\hline & XPF-ERCC1?-BTBD12 & XPF & $\mathrm{N} / \mathrm{D}$ & \\
\hline & MUS81-EME1?-BTBD12 & XPF & $\mathrm{N} / \mathrm{D}$ & \\
\hline & SLX1-BTBD12 & UIY-YIG & HR, ICL, DSBR & $\begin{array}{l}\text { (Andersen et al. 2009; Fakairi et al. 2009; } \\
\text { Svendsen et al. 2009; Munoz et al. 2009) }\end{array}$ \\
\hline
\end{tabular}

$N / D$ not determined, NER nucleotide excision repair, SSA single-strand annealing, MTS mating-type switching, $H R$ homologous recombination, $R F$ replication fork support, $I C L$ interstrand crosslink repair, $r D N A$ ribosomal DNA maintenance, $D S B R$ double-strand break repair

On page 117, left column Line 44: replace 'strong a preference' with 'a strong preference'. The correct sentence must read

There is a wide consensus that Mus81-Mms4/EME1 endonucleases from all organisms studied have a strong preference for nicked junction substrates over classical HJ substrates.

On page 117, right column Line 34 replace 'Rad2/XPF' with 'Rad2/XPG'
On page 117, right column Line 37: Replace 'a 381-amino-acid C-terminal truncation' by: 'an approximately $60 \mathrm{kDa}$ N-terminal fragment.'

The correct sentence must read

The RuvC-like activity in human cells was determined to be dependent on the catalytic activity of the Rad2/XPG endonuclease HsGEN1 (Ip et al. 2008). HsGEN1 was isolated by column fractionation and identified by mass spectroscopy as an approximately $60 \mathrm{kDa} \mathrm{N}$-terminal fragment. 